\title{
Influencia de la Televisión Violenta en Niños de una Escuela Pública de Bogotá, Colombia
}

\author{
ISABEL PÉREZ-OLMOS, ÁNGELA MARÍA PINZÓN, RODRIGO \\ GONZÁLEZ-REYES y JULIANA SÁNCHEZ-MOLANO
}

Recibido 15 Diciembre 2004/Enviado para Modificación 31 Diciembre 2004/Aceptado 31 Enero 2005

\section{RESUMEN}

Objetivos Conocer el impacto en niños escolares ocasionado por una película violenta y otra no violenta.

Métodos En una escuela pública de Bogotá, 125 estudiantes de primaria respondieron una encuesta sobre la violencia de su vida diaria y su actitud ante ella. Con intervalos de quince días se les presentó la película 1, violenta, y la película 2, no violenta. Después de cada película, los niños realizaron la prueba del dibujo de familia, escribieron su opinión sobre la película, y respondieron la encuesta.

Resultados En la encuesta inicial, $23,6 \%$ de los niños refirió respuestas violentas cuando son agredidos, 39,8\% reportó algún tipo de violencia intrafamiliar y el $19,5 \%$ se identificó con figuras violentas. Los varones fueron más propensos a responder violentamente ante la agresión y a identificarse con figuras violentas $(p=0,004)$. Después de la película violenta, un mayor porcentaje de niños se excluyó a si mismo en el dibujo de familia, comparado con los que lo hicieron después de la película no violenta, Razón de Desventaja (RD) 2,55 (Intervalo de Confianza $95 \%$ (IC) 1,22-5,43, p=0,01). El dibujo de familia de la película violenta presentó mayores frecuencias de signos emocionales que el dibujo 2, RD 3,13 (IC 1,35-7,52, p=0,0053), y más signos de agresividad, RD 2,55 (IC 1,22-5,43, p=0,010).

Conclusiones La prueba del dibujo de familia permite evidenciar el impacto inmediato de la televisión. La violencia televisiva afecta negativamente a los niños y debería ser evitada.

Palabras Clave: Televisión, niño, violencia, pruebas, psicología, Colombia (fuente: DeCS, BIREME) 


\section{ABSTRACT \\ Influence of violent TV upon children of a public school in Bogotá, Colombia}

Objective To evaluate the impact that a violent and a non-violent movie may cause on scholars.

Methods In Bogotá, 125 public primary school students were surveyed, applying a questionnaire to learn both about their daily life violence and their attitude towards it. Two weeks later, they were shown one violent movie, and two weeks later a non-violent one. Children were asked to draw their families, express their opinions and answer a questionnaire after each movie. Results The initial survey showed that $23,6 \%$ of the children reported violent responses when they were offended, 39,8 \% reported some kind of familiar violence and 19,5\% identified themselves with a violent figure. Boys were more prone to respond violently when offended and to identify themselves with a violent figure than girls $(p=0,004)$. Compared with the non-violent movie, a greater percentage of children excluded themselves from the family drawing after watching the violent movie (Odds Ratio (OR): 2.55; 95 $\%$ Interval Confidence $(95 \% \mathrm{Cl}) 1.22-5.43, \mathrm{p}=0,01)$. The family drawing after the violent movie also showed more emotional signs (OR: 3.13; $95 \% \mathrm{Cl}$ : 1.35-7.52; $p=0,0053$ ) and more aggressive signs (OR: $2.55 ; 95 \% \mathrm{Cl}: 1.22$ $5.43 ; p=0,01)$ than the family drawing after the non-violent movie.

Conclusions The family drawing test showed the immediate impact of television. Television violence negatively influences kids and should be avoided.

Key Words: Television, child, violence, psychological tests, Colombia (source: $\mathrm{MeSH}, \mathrm{NLM}$ ).

$\mathrm{E}$ n las últimas décadas, la sensibilidad social frente a la violencia en los medios de comunicación, en especial la televisión, ha aumentado significativamente. Diferentes investigadores han demostrado que las imágenes emitidas por estos medios ejercen una influencia importante sobre la conducta del individuo especialmente si es infante (1-3).

El desarrollo psíquico es un proceso de complejidad gradual que establece las bases de la conducta en la infancia y adolescencia así como en la edad adulta (4). El ambiente influye en el desarrollo físico así como en el psicológico. Las características del pensamiento infantil en proceso de maduración y el cual inicialmente es predominantemente concreto $(1,4)$, favorecen esta influencia. La edad entre los 8 a 12 años es crítica debido a que no existe una clara diferencia entre lo real y lo imaginario, y está favorecida la invención, imitación, e identificación con héroes agresivos de fantasías heroicas o violentas, lo que a su vez, sirve para propiciar respuestas violentas 
en situaciones de la vida real. Esta situación se explica también por el predominio del pensamiento pre-lógico característico del infante $(1,5)$. Los niños de 11 a 12 años comienzan a discriminar entre lo real y lo ficticio ya que han desarrollado un pensamiento más elaborado que incluso les permite hacer críticas de los contenidos violentos $(1,4)$.

El mundo mental del niño es maleable, influenciable y susceptible a los cambios por lo que requiere un importante refuerzo emocional y educativo del entorno, especialmente de la familia, y en general de la sociedad. Si durante el periodo de maduración alguno de estos elementos falla, se facilitarán otras influencias externas que pueden ser negativas.

Dentro de estas últimas se encuentran los medios masivos de comunicación principalmente la televisión. Según datos de Estados Unidos, un 98\% de los hogares norteamericanos cuenta con por lo menos un televisor (6), estando estos encendidos por lo menos durante 7 horas al día (7). Esto ha contribuido a la modificación enorme tanto del estilo de vida como de los hábitos de interacción familiar.

El fenómeno de los contenidos violentos en la televisión ha sido investigado mediante dos enfoques, uno positivista y otro de tipo hermenéuticocomprensivo. El primer enfoque (Paradigma positivista) defendido por autores como Bandura y Walters, Berkowitz y Friederich \& Huston, sostiene que la televisión es transmisora de comportamientos violentos que propician un aprendizaje modelado y condicionado de pautas agresivas $(1,8)$. Los niños con alto grado de exposición a este medio pueden exhibir una alta incidencia de hostilidad al imitar la agresión de la cual ellos mismos son testigos (8).

El segundo punto de vista (Paradigma hermenéutico-comprensivo), defendido también por una amplia cantidad de autores, adopta una mirada integradora entre lo psíquico y lo socio-cultural (1). Los efectos negativos de la televisión violenta en los niños incluyen: a) tener menor sensibilidad al dolor (2), b) comportamientos agresivos, c) la posibilidad de emplear la violencia como mecanismo para resolver conflictos, d) victimizar a otros y e) desarrollar pensamientos y creencias acerca del mundo como un lugar malo y tenebroso (9).

Las pautas publicitarias, los aspectos económicos, demográficos y sociales que hacen que un individuo vea televisión en mayor o en menor grado que otro, el tipo de personalidad, las características emocionales y el contexto cultural de una determinada persona o grupo de personas entre muchos 
otros, son factores que pueden influenciar el impacto sobre las actitudes del televidente (9).

Los niños están creciendo bajo la influencia de un ambiente altamente televisivo. Se comienza la relación con el televisor cada vez desde una edad más temprana y es frecuente el uso de la televisión como "niñera", mientras los padres o cuidadores realizan otras actividades.

Por fortuna, la televisión no sólo tiene potenciales efectos negativos en los niños, también puede tener efectos positivos. Se han publicado estudios que exponen los posibles efectos benéficos de la televisión (3). Rushton, afirma que los niños presentan respuestas emocionales cuando ven televisión y que los seres humanos aprenden a hacer las cosas por medio de la observación (10). El niño puede imitar conductas favorables presentadas en la televisión como el juego amistoso y la resolución pacífica de conflictos, el altruismo, el auto-control, la anti-estereotipación, y en general puede modelar positivamente sus relaciones sociales (11).

Se ha encontrado que la televisión ejerce una mayor influencia en los niños que la radio, al parecer porque ellos tienen una mejor respuesta frente a historias que contienen imágenes gráficas $(12,13)$.

A pesar de que múltiples estudios han mostrado que la televisión violenta influye negativamente en los niños, ellos continúan siendo expuestos a programas de televisión con contenidos violentos. Se hace necesario profundizar en el conocimiento de este fenómeno con el fin de regular adecuadamente los hábitos y la exposición televisiva de los niños.

Para enriquecer el abordaje científico del complejo tema del impacto de la televisión violenta en los niños, esta investigación usa métodos cuantitativos combinados con métodos cualitativos (14-17). Dentro de los instrumentos cualitativos se encuentran las pruebas psicológicas proyectivas. Éstas reflejan las percepciones que los sujetos tienen del mundo y no pueden ser objeto de una estandarización completa, por lo que no cumplen con los estándares positivistas convencionales de confiabilidad y validez (18-20) y se rigen por los criterios en que se apoya la investigación cualitativa.

El dibujo de familia es un test psicológico proyectivo con poca estructuración que permite la proyección global, consciente e inconsciente, de la personalidad del dibujante y a la vez suficiente estructuración que permite la comparación entre sujetos (18). 
Los ítem evolutivos del dibujo de figura humana establecen el nivel de maduración cognitiva del dibujante (19). Los ítem emocionales difieren de los evolutivos al reflejar las ansiedades, preocupaciones y actitudes de los dibujantes. Deben cumplir los criterios de validez clínica, es decir diferenciar a los niños con y sin problemas emocionales, ser inusuales en los niños sin problemas psiquiátricos (frecuencia menor del 16\%) y no deben estar relacionados con la edad y la maduración del niño $(19,21)$.

Algunos autores establecen que el test del dibujo de la figura humana puede ser usado como prueba complementaria en la evaluación de la inteligencia infantil, por su validez y fácil aplicación y también puede ser usado como prueba de tamización en grupos infantiles (22).

\section{MATERIALES Y MÉTODOS}

La población estudiada se compuso de 125 niños, alumnos de segundo hasta quinto de primaria de una escuela pública de Bogotá.

De la programación diaria del horario infantil de los canales colombianos en la época de la realización de la investigación se seleccionaron dos películas infantiles, una película de contenido violento y otra no violenta. A continuación se resumen muy brevemente cada una de las películas.

Película violenta

Un mortal y sangriento combate por salvar la Tierra se desarrolla entre las poderosas y maléficas fuerzas de un líder negativo y sus cinco guerreros contra el héroe y sus amigos. Al final, el héroe se ve obligado a arriesgar la vida de su hijo, para poder destruir al ambicioso y malvado villano.

Película no violenta

Aventuras y desventuras de un pequeño niño, que hará lo imposible por recobrar el protagonismo que le ha quitado su recién llegado hermano pequeño. Junto a sus amigos continuará disfrutando de las más variadas aventuras.

El orden de presentación de las películas, primero la violenta y quince días después, la no violenta, busca que el impacto de la película violenta, objeto del presente estudio, se pudiese comparar con el posterior impacto de la película no violenta. Si se invirtiese este orden las respuestas de los niños favorecerían al estímulo no violento en primera instancia. Con esta secuencia además se buscó que la última exposición realizada fuera la no violenta, 
para proteger a los menores de recibir un estímulo negativo al final del estudio por parte de los investigadores.

Instrumentos Utilizados

Con asesoría metodológica y psicológica, se diseñó una encuesta de 10 preguntas, ilustradas con figuras de fácil identificación para los niños. La encuesta buscaba establecer las actitudes, respuestas agresivas, identificación con figuras violentas y la violencia intra-familiar de los niños. Se hizo una prueba piloto en una escuela pública similar a la escuela seleccionada, hasta definir la encuesta definitiva.

Se utilizó el test del dibujo de familia y los parámetros del dibujo de la figura humana, pruebas psicológicas proyectivas, para evidenciar el efecto de las películas en los niños y para observar diferencias entre el impacto de la película violenta y la no violenta. Los dibujos de familia fueron analizados por una psiquiatra sicoanalista epidemióloga y dos médicos previamente entrenados.

En cada dibujo se determinaron los signos de maduración cognitiva, indicadores emocionales y de agresividad, el tipo de trazo y sector de la página utilizado, el marco sensorial y racional del dibujo, la estructura e interacción familiar, los signos de rivalidad fraterna, la desvalorización o ausencia de cada miembro de la familia. La inclusión de dibujos adicionales, animales y las manifestaciones gráficas de algunos mecanismos de defensa $(18,19)$.

La recolección de la información se realizó en tres momentos. En el primero se aplicó la encuesta a los niños. En el segundo momento, 2 semanas más tarde, los niños observaron la película violenta, e inmediatamente después elaboraron el dibujo de familia, escribieron su opinión de la película y respondieron la encuesta. En el tercer momento, realizado quince días después, con el fin de eliminar el sesgo de recuerdo, se transmitió la película no violenta, y se repitió el mismo proceso de la película anterior. De esta manera se podrían comparar las respuestas de cada niño ante cada película.

Recolección de la Información

Las fuentes de la información fueron las encuestas diligenciadas, los dibujos de familia realizados y las opiniones acerca de las películas escritas por los niños del estudio.

Análisis Cualitativo y Cuantitativo

Encuestas 
Las respuestas de las encuestas fueron tabuladas y digitadas en una base de datos por dos personas distintas, las bases de datos fueron comparadas para evitar errores. Las preguntas fueron divididas en tres grupos para la interpretación de las mismas; grupo 1, respuestas frente a la agresión; grupo 2, preguntas del ambiente familiar y grupo 3, auto imagen del niño/a e identificación con figuras violentas. La información fue analizada utilizando los programas estadísticos SPSS y STATA.

Prueba del dibujo de familia y opiniones acerca de las películas

La información recolectada a partir de la pruebas del dibujo de familia y de las opiniones de los niños fue registrada por cada investigador en forma cualitativa y posteriormente fue codificada y registrada en una base de datos de Excel. La información obtenida por cada evaluador fue comparada y el consenso entre ellos fue tabulado en la base de datos, se obtuvieron las proporciones de estas variables.

Para comparar el impacto de las películas en cada niño, a través de los dibujos de familia, solamente se utilizaron los dibujos de familia que 91 niños, que asistieron a ambas películas, hicieron después de cada una. Se crearon dos variables que recogían la eliminación del dibujante o de algún otro miembro de la familia, la existencia de signos emocionales y de agresividad en los dibujos correspondientes a cada película. Estos datos fueron evaluados con la Prueba estadística de McNemar para La Significación de los Cambios. El análisis se hizo con el programa SPSS y STATA.

La participación en el estudio fue voluntaria. El protocolo del estudio fue aprobado por el comité de ética de la Facultad de Medicina de la Universidad del Rosario.

\section{RESULTADOS}

Características generales de los niños estudiados

La distribución de los niños por cursos fue: 28 en segundo de primaria, 32 en tercero, 31 en cuarto y 31 en quinto de primaria. Los 121 niños cuyos dibujos de familia se analizaron tenían edades entre 7 y 15 años. La edad promedio fue 9,6 años (desviación estándar, 1,77 años). La edad mas frecuente fue 9 años con un 25,6 \% del grupo, seguida de 10 y 8 años respectivamente. El $50 \%$ del grupo tenía máximo 9 años de edad. El 62 \% eran hombres (n=75). 
La comparación de cambios en los dibujos de familia 1 y 2 y en las opiniones sobre cada película, en cada niño, sólo se pudo hacer en 91 niños que completaron la información correspondiente posterior a las dos películas.

Los resultados se presentan distribuidos así: 1 . Resultados de la encuesta basal ( $\mathrm{n}=123$ niños). 2. Encuestas posteriores a la película 1, violenta, $(\mathrm{n}=123)$, y a la película 2, no violenta, $(\mathrm{n}=96)$. 3 . Dibujos de familia posteriores a la película $1(\mathrm{n}=113)$ y posteriores a la película $2(\mathrm{n}=102)$, y comparación entre los mismos $(n=92)$. 4. Análisis de las opiniones de los niños sobre las películas $(\mathrm{n}=121)$. El número de niños en cada análisis corresponde a los que asistieron y participaron voluntariamente a la actividad correspondiente.

\section{Encuesta Basal}

Fue respondida por 123 niños de los cuales el $61 \%$ eran hombres. El 54,5 \% de los niños incluyó algún tipo de violencia en las respuestas de la encuesta basal, encontrándose que el 23,6 \% de ellos responderían de forma violenta frente a la agresión, el 39,8 \% refirió algún tipo de violencia intrafamiliar y el 19,5 \% se identificó a sí misma/o con figuras violentas.

Al comparar la identificación con figuras violentas y las respuestas violentas con el género de los niños, se encontró que los niños son más propensos a responder violentamente frente a la agresión y a identificarse con figuras violentas que las niñas; esto realizando un análisis estadístico descriptivo de tendencias (Tabla 1).

\section{Encuestas posteriores a cada película}

En primer lugar se realizó un análisis de antes y después de las respuestas de 93 niños que diligenciaron las dos encuestas, mediante el test pareado de McNemar. En este análisis no se encontraron diferencias significativas. Posteriormente se analizaron 123 encuestas diligenciadas después de la primera película y 96 diligenciadas después de la segunda y se calculó el porcentaje de respuestas agresivas a cada pregunta. Estos porcentajes se pueden evidenciar en la tabla 1.

En general se puede concluir que no se presentaron diferencias en las preguntas relacionadas con respuesta a la agresión; que en las preguntas relacionadas con el ambiente familiar se presentaron más respuestas agresivas después de observar la película no violenta y que en las preguntas relacio- 
nadas con identificación de si mismo se encontraron más respuestas agresivas después de observar la película violenta.

Tabla 1. Encuestas. Comparación de respuestas según tipo de película observada

\begin{tabular}{|c|c|c|c|}
\hline Especificación de la Pregunta & Tipo & $\begin{array}{c}\text { Película } \\
\text { violenta } \\
\% \\
\end{array}$ & $\begin{array}{c}\text { Película } \\
\text { no } \\
\text { violenta \% }\end{array}$ \\
\hline $\begin{array}{c}\text { ¿Si dos niños están peleando tu que } \\
\text { haces? }\end{array}$ & $\begin{array}{l}\text { Respuesta frente a la } \\
\text { agresión }\end{array}$ & 26,0 & 26,0 \\
\hline ¿Cuál se parece más a tu papá? & Ambiente familiar & 22,8 & 24,6 \\
\hline $\begin{array}{c}\text { ¿Si un niño te dice una grosería, tú } \\
\text { que haces? }\end{array}$ & $\begin{array}{l}\text { Respuesta frente a la } \\
\text { agresión }\end{array}$ & 4,9 & 5,3 \\
\hline $\begin{array}{c}\text { ¿Cómo cual de estos personajes te } \\
\text { gustaría ser?* }\end{array}$ & Identificación de si mismo & 16,0 & 7,3 \\
\hline ¿Con tus hermanos, tu que haces? & Ambiente familiar & 3,2 & 4,2 \\
\hline $\begin{array}{c}\text { ¿Si te portas mal, tú mamá que } \\
\text { hace? }\end{array}$ & Ambiente familiar & 25,2 & 30,2 \\
\hline ¿Cuál es tu juguete favorito? & Identificación de si mismo & 21,1 & 18,7 \\
\hline Las personas que viven contigo...* & Ambiente familiar & 2,4 & 8,3 \\
\hline $\begin{array}{l}\text { ¿Si una niña te quita algo, tu que } \\
\text { haces? }\end{array}$ & $\begin{array}{l}\text { Respuesta frente a la } \\
\text { agresión }\end{array}$ & 18,7 & 17,7 \\
\hline
\end{tabular}

Dibujos de Familia

Asistieron a las dos películas e hicieron los dibujos de familia, el 76 \% (n= 92); faltaron a la primera película el 7,4 \% ( $\mathrm{n}=9)$, y faltaron a la segunda película el 16,5 \% (n=20).

Signos de Inmadurez Cognitiva

Al comparar los signos de inmadurez de cada niño en el dibujo 1 (posterior a la película violenta) y el 2 (película no violenta) se encontró que un 13,2 \% $(\mathrm{n}=16)$ tenía signos de inmadurez sólo en el primer dibujo y el 9,9\% $(\mathrm{N}=12)$ sólo en el dibujo 2 (Figura 1).

El 51,2 \% (n= 62) de los niños no presentó signos de inmadurez cognitiva para su edad, y el 25,6 \% (n=31) mostró algún grado de inmadurez en ambos dibujos (Figura 2). 
Figura 1. Características de Dibujos de Familia Posteriores a Película violenta y no violenta. Bogotá, 2002

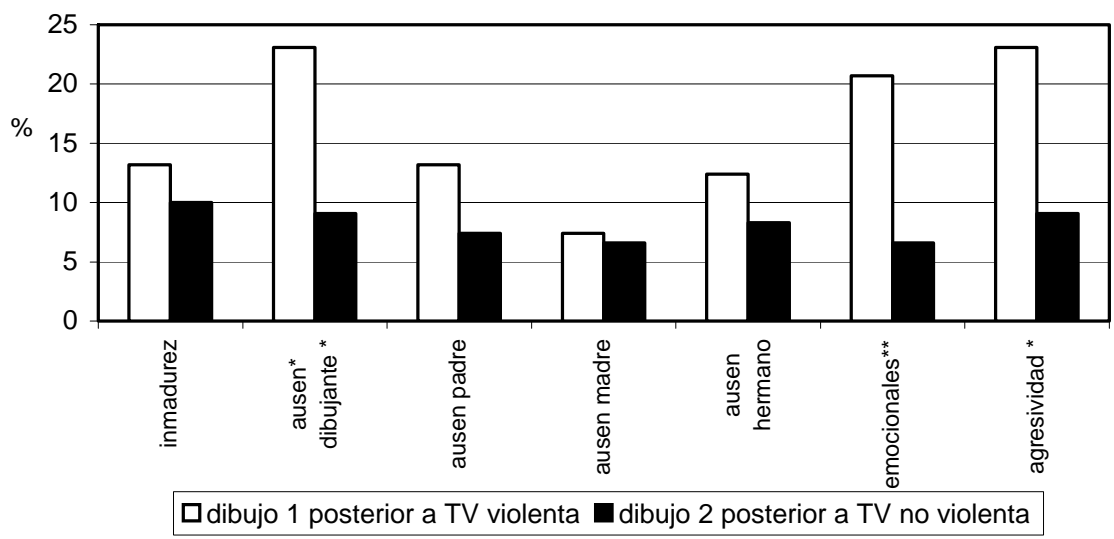

$R D$ * $P=0.01 ; R D$ ** $P=0.0053$

Figura 2. Signos de inmadurez cognitiva según edad en dibujos de familia posteriores a películas. Bogotá, 2002

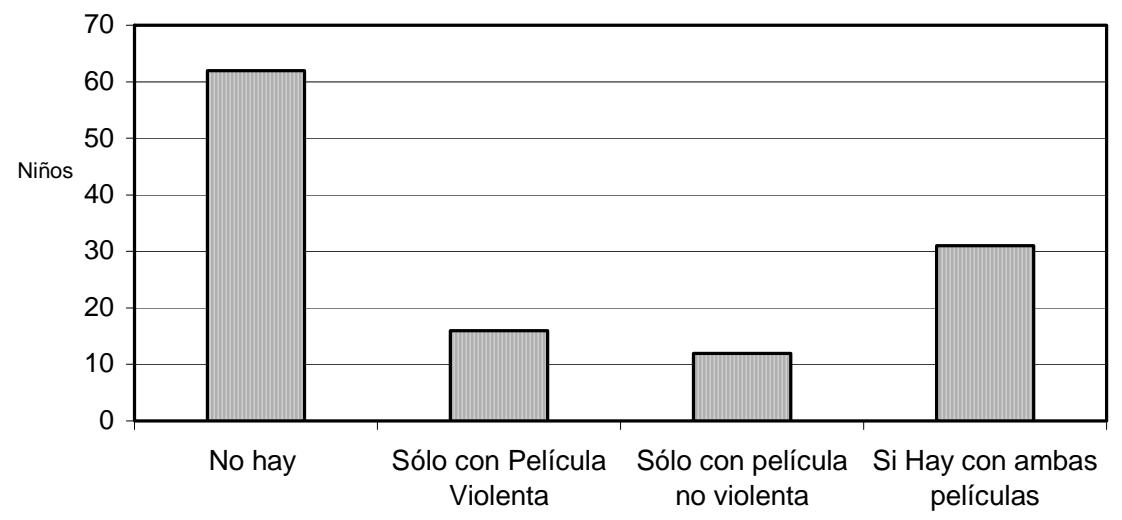

Ausencia de miembros de la familia en los dibujos

Ausencia del dibujante 
Los niños se incluyeron a si mismos en la familia en el 52,1 \% $(n=63)$ de los dibujos y se excluyeron de ambos dibujos en el $15 \%(n=18)$. Sólo en un dibujo no se pudo identificar al dibujante $(0,8 \%)$. El 23,1 \% (n=28) de los niños se excluyó a sí mismo sólo en el primer dibujo, mientras que esta exclusión ocurrió sólo en el segundo dibujo en el 9,1 \% ( $\mathrm{n}=11)$. Razón de Disparidad (RD) 2,55 (IC 1,22-5,43, P=0,01).

Ausencia del padre en el dibujo

La figura del papá fue incluida en ambos dibujos de familia en el 61,2 \% $(n=74)$ y fue excluida de ambos dibujos en el $16,5 \%(n=20)$. En dos dibujos no se pudo identificar al padre $(1,7 \%)$. La figura del papá se excluyó sólo del primer dibujo en el 13,2 \% ( $\mathrm{n}=16)$. Mientras que se excluyó sólo en el segundo dibujo en el 7,4 \% ( $n=9)$ (Figura 1$)$.

Ausencia de la madre en el dibujo

Los dibujos de familia incluían a la mamá en el 77 \% (n=93) y la excluían en el 7,4 \% (n=9). En dos casos no se pudo identificar a la madre (1,7 \%). El $7,4 \%(n=9)$ de los niños excluyó a la mamá solo en el primer dibujo, mientras que el 6,6 \% $(n=8)$ lo hizo sólo en el segundo dibujo (Figura 1).

Exclusión de hermanos o hermanas del dibujo

Los hermanos o hermanas fueron incluidos en el $58 \%(n=70)$ de los dibujos de familia. Algún hermano o hermana fue excluido en ambos dibujos, a pesar de contar con ellos en el $19 \%(n=23)$. En tres casos no se pudo identificar a los hermanos (2,5\%). Se excluyó a algún hermano/a solamente en el primer dibujo en el $12,4 \%(\mathrm{n}=15)$; mientras que esto ocurrió sólo en el segundo dibujo en el 8,3\% (n=10) (Figura 1).

\section{Elementos emocionales}

No se encontraron signos emocionales en el 2,5 \% $(n=3)$ de los dibujos. Hubo algún tipo de signo emocional en el 70,2 \% $(\mathrm{n}=85)$ de los dibujos. Se encontraron signos emocionales sólo en el primer dibujo en el 20,7 \% ( $\mathrm{n}=25)$ y sólo en el segundo dibujo en el 6,6 \% $(\mathrm{n}=8)$ de los niños. RD 3,13 (IC 1,35-7,52, $\mathrm{P}=0$,0053) (Figura 1).

Uno de los elementos emocionales analizados fue la adición de dibujos diferentes a la familia solicitada. No hubo imágenes adicionales en el 42,1 \% $(n=51)$ de los dibujos. Los niños incluyeron imágenes adicionales en el $34 \%$ 
$(n=41)$ de los dibujos. Esto ocurrió sólo en el dibujo 1 en el $14 \%(n=17), y$ lo mismo ocurrió sólo en el dibujo 2, en el $10 \%(n=12)$.

\section{Agresividad}

No hubo signos agresivos en el 12,4 \% $(n=15)$ de los dibujos. Se encontraron elementos de agresividad en el 55,4 \% $(n=67)$ de los dibujos (Figura 3). Hubo elementos de agresividad sólo en el primer dibujo en el 23,1 \% ( $n=28)$ de los niños y esto ocurrió sólo en el dibujo 2, en el 9,1 \% $(n=11)$ de los niños. RD 2,55 (IC 1,22-5,43, P=0,010) (Figura 1).

Figura 3. Signos de agresividad en dibujos de familia posteriores a películas. Bogotá, 2002

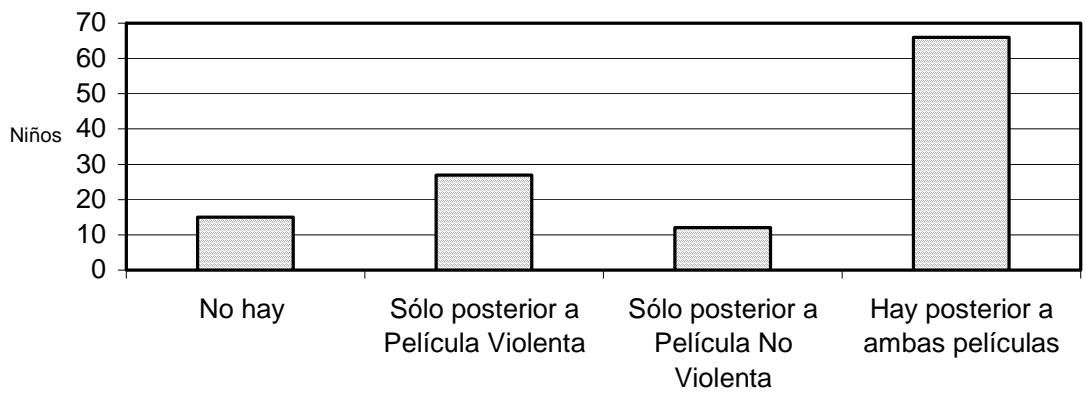

Resumen comparativo del dibujo 1 (película violenta) y el dibujo 2 (no violenta)

En el dibujo uno, el 89,3 \% (n=108), tuvo algún tipo de manifestación emocional, agresiva o de inmadurez cognitiva. En el dibujo dos, el $62 \%$ $(n=75)$, manifestó algún signo emocional, agresivo o de inmadurez cognitiva. En resumen, el impacto registrado en el dibujo de familia posterior a la película violenta fue mayor que el registrado luego de la película no violenta.

El $19 \%$ de los dibujos ( $\mathrm{n}=23$ ) no tuvo manifestaciones que pudieran atribuirse a impacto inmediato de alguna de las películas. El porcentaje de no impacto fue mayor en el dibujo 2, (película no violenta), que en el dibujo 1 (película violenta).

Se aplicó la prueba estadística de McNemar, para la significación de los cambios, a los datos de impacto manifiesto en el dibujo 1 y 2 . Se encontró 
una diferencia estadísticamente significativa en la comparación, ya que, mientras 18 niños presentaron signos agresivos o emocionales sólo en el dibujo 1 sin presentarlos en el 2, sólo un niño presentó manifestaciones emocionales y agresivas en el segundo dibujo sin presentarlos en el dibujo 1 (Tabla 2).

Finalmente es importante resaltar que un alto porcentaje (75 \%) de niños $(\mathrm{n}=68)$ presentó en ambos dibujos manifestaciones emocionales, agresivas o de inmadurez cognitiva (Tabla 2).

Tabla 2. Comparación dibujos de familia. Película violenta y no violenta

\begin{tabular}{|c|c|c|c|c|}
\hline \multirow{2}{*}{\multicolumn{2}{|c|}{$\begin{array}{c}\text { Signos emocionales y agresivos } \\
\text { Dibujos de Familia }\end{array}$}} & \multicolumn{2}{|c|}{$\begin{array}{c}\text { Dibujo Familia } 2 \\
\text { película no violenta }\end{array}$} & \multirow[b]{2}{*}{ Total } \\
\hline & & no tiene & si tiene & \\
\hline \multirow{2}{*}{$\begin{array}{l}\text { Dibujo Familia } 1 \\
\text { película violenta }\end{array}$} & no tiene & 4 & 1 & 5 \\
\hline & si tiene & 18 & 68 & 86 \\
\hline \multicolumn{2}{|c|}{ Total } & 22 & 69 & 91 \\
\hline
\end{tabular}

Opinión de la película

La opinión de los niños fue favorable para ambas películas en el $48 \%$ $(\mathrm{n}=58)$. Las opiniones desfavorables o ambivalentes sobre ambas películas se presentaron en el 9,1\% $(\mathrm{n}=11)$, todos estos niños eran mayores de 9 años. Opiniones negativas sólo de la película 1 (violenta) ocurrieron en el 32,2 \% ( $n=39)$, y sólo para la película 2 (no violenta) en el 10 \% ( $\mathrm{n}=12)$ de los niños.

En los escritos se pudo identificar algún grado de dislexia en el 7,4 \% $(\mathrm{n}=9)$ de los niños. También se encontraron errores frecuentes de ortografía en el 56,2 \% ( $n=68)$ de los escritos de los niños.

\section{DISCUSIÓN}

Mas de la tercera parte de los niños de este estudio reportaron algún tipo de violencia intra-familiar y casi la cuarta parte refirió respuestas violentas frente a la agresión; este hallazgo es consistente con las cifras de violencia intra familiar reportadas en las estadísticas locales y nacionales colombianas. Además, el $20 \%$ de los niños estudiados se identificó a si mismo con figuras violentas y esto concuerda con el uso frecuente de la agresión y violencia 
que ha caracterizado nuestro contexto cultural. Esta situación ha predominado en forma creciente en Colombia durante las últimas décadas aunque hace 40 años era ya motivo de alarma en la Salud Publica $(23,24)$. Sin embargo, este fenómeno no ha recibido la atención requerida por parte de las instituciones sociales y los diferentes sectores de la comunidad. Otro resultado importante es que los varones se identificaron con figuras violentas y tenían respuestas agresivas con mayor frecuencia que las niñas, este hallazgo también se ha descrito en la literatura científica sobre el tema, tanto en el nivel nacional como internacional $(23,25)$.

En la revisión bibliográfica realizada se encontraron pocos estudios que exploraran el efecto inmediato de los contenidos violentos en la tele-audiencia infantil. Esta investigación buscó reunir evidencias en ese sentido y comparar con el impacto de una película no violenta. Nuestros resultados son consistentes con reportes de otros investigadores acerca de los efectos de la violencia televisiva sobre la cognición, la emoción y la conducta infantil (3,26-28). Estos estudios confirman efectos negativos a largo plazo en la conducta del infante por exposición repetida a películas violentas. La teoría de diferentes autores es que se genera un comportamiento agresivo en los niños expuestos (29-31).

Sólo se encontró una investigación con evidencia contraria a la planteada; Feshback y Singer (32), concluyen que ver televisión violenta durante 6 semanas tiende a disminuir las respuestas agresivas en la audiencia juvenil mientras que observar películas no violentas durante el mismo periodo aumenta la probabilidad de conductas agresivas. Los hallazgos fueron explicados con la teoría de la catarsis de sentimientos y pensamientos agresivos por liberación de estos en la relación visual con el acto violento observado.

Nuestro estudio presenta evidencias del impacto inmediato de la televisión violenta en los niños, consistente en frecuencias significativamente mayores de auto-exclusión del dibujante en el dibujo familiar posterior a la película violenta en comparación con el dibujo posterior a la película no violenta. También hubo mayores frecuencias significativas de signos emocionales y agresivos en el dibujo posterior a la película violenta. Estos hallazgos evidencian el efecto cognitivo y emocional que el drama violento ocasiona en el niño, y también concuerda con el impacto descrito por Aran y colaboradores, (1).

La psico-dinamia permite comprender la autoeliminación del dibujante en el grupo familiar y está relacionada con el desarrollo de la personalidad y del si-mismo (self). Durante este proceso se implementan sistemas de autocon- 
trol de la conducta y en general de la impulsividad características del periodo infantil. La autoeliminación del dibujante puede ser entendida en términos de regresión psicológica con predominio de la actuación irreflexiva propia de las etapas tempranas del funcionamiento mental, (acting out, DSM IV, 1995), (33).

El niño identificado con los personajes y héroes agresivos cuyo éxito se reduce a lograr la destrucción del otro, deja de percibirse a si-mismo o se evade de su auto-percepción en la realidad de su entorno inmediato. Estas dinámicas psicológicas y conductuales también permitirían explicar y comprender porqué hubo mayor frecuencia de signos de inmadurez cognitiva en los dibujos realizados luego de la película violenta.

Hubo una tendencia, aunque no alcanzó el nivel de la significación estadística, hacia frecuencias mayores de supresión de la figura del padre y hermanas o hermanos luego de la película violenta, sin embargo esta requeriría ser evaluada en una muestra de niños más grande, para verificar si se alcanza el nivel de significación estadística.

Los resultados presentados concuerdan con los efectos señalados por Bandura, Mares, Delores, Kremar y Robinson et al y otros autores debidos a la exposición visual a los medios violentos. Es importante señalar que la figura de la madre tuvo igual frecuencia de preservación en los dibujos de familia realizados después de ambas películas. Este hecho confirma que es justamente la figura de la madre la que brinda a los niños el apoyo afectivo necesario para hacer frente a situaciones amenazantes de la vida diaria.

Los resultados también evidencian que en los grupos de menor edad, las consideraciones éticas no están presentes en el discurso espontáneo (1). También es interesante señalar el hallazgo de que algunos niños que rechazaron la violencia de la primera película tenían dibujos con signos de mayor maduración cognitiva que aquellos que no lo hicieron.

En resumen, los hallazgos de este estudio así como la revisión bibliográfica realizada indican que la exposición de los niños a situaciones de violencia, reales o virtuales, en la televisión y en otros medios masivos de comunicación, e incluso en la nuevas realidades virtuales, genera un impacto mental (emocional, cognitivo, conductual) del cual pueden encontrarse evidencias tanto en forma inmediata como a largo plazo. Esto confirmaría lo que múltiples autores ya han señalado, esta exposición influye en el comportamiento de los niños al favorecer las conductas violentas. 
Las múltiples evidencias y la contra-evidencia presentadas señalan la necesidad de continuar profundizando en el estudio del tema y en la exploración de las relaciones entre el estimulo audiovisual violento y no violento, el tipo de drama, las características personales de la tele audiencia, especialmente la infantil y juvenil, y de otros factores que producen efectos a corto y largo plazo.

Es importante señalar que aunque los instrumentos utilizados no reúnen los porcentajes de validación esperados en el método científico positivista, en el análisis comparativo que se hizo del efecto de cada película, cada niño fue su propio control, es decir, las manifestaciones de cada niño no se compararon con las de otros niños sino con su propia respuesta en otro momento y posterior a otro estímulo, mediante el uso de los mismos instrumentos: la encuesta, el dibujo de familia y la opinión escrita. Las comparaciones evaluaron el impacto inmediato de cada película en cada niño, se compararon sus propias respuestas en el mismo instrumento luego de la exposición a la película violenta y a la no violenta.

Debe también realizarse un estudio a mayor profundidad en los niños y sus familias, ya que, por un lado, una cuarta parte de ellos presentaron, signos de inmadurez cognitiva con relación a su edad cronológica. Tres cuartas partes de ellos presentaron signos emocionales y la mitad de ellos, agresivos. Estas expresiones, no atribuibles directamente a las películas puesto que se presentaron después ver las dos, pueden corresponder a manifestaciones de situaciones psicológicas y de la vida familiar de los niños y requieren ser estudiadas para poder implementar intervenciones dirigidas a mejorar la calidad de vida de estos niños y su convivencia con sus familias.

Por otro lado, la frecuencia de signos de dislexia encontrados en los escritos concuerda con la prevalencia descrita en la literatura científica (34). Se identificaron también manifestaciones del impacto del drama del nacimiento del hermano de la segunda película, algunos dibujos de familia luego de esta película mostraron al dibujante más cercano a la madre o a ésta acompañada de un bebé o incluyeron bebés en la escena familiar, características no evidenciadas en los dibujos de la primera película.

El dibujo fue el medio usado por los niños, más frecuentemente que la opinión escrita, para expresar el impacto negativo de la película, lo que muestra su utilidad para el estudio de estos aspectos, especialmente en los niños más pequeños. 
Finalmente, debemos insistir en la importancia que debe prestarse a la influencia negativa de los medios violentos sobre los niños para que tanto los padres de familia, los educadores, el personal de salud y la comunidad en general, tengan en cuenta este hecho y asuman un actitud pro-activa a favor de auspiciar el buen desarrollo de los niños, facilitando la exposición recreativa y regulada de los niños con proyecciones educativas que estimulen modelos positivos y de efecto benéfico sobre su desarrollo y salud mental.

\section{Limitaciones del estudio}

El diseño descriptivo de la investigación no puede ser demostrativo e impide tener una certeza acerca de las relaciones causales entre la exposición televisiva realizada y las respuestas de impacto de los niños. Las conclusiones planteadas sólo conducen a plantear una hipótesis de impacto negativo de la televisión violenta en los niños que deberá ser falseada posteriormente con otro tipo de investigación. Un nuevo diseño experimental con grupo control podría probar esta hipótesis y le aumentaría validez al estudio. Debido a no contar con un instrumento en español validado para determinar tanto la exposición a la violencia como la conducta violenta cotidiana de los niños del estudio, se creó una encuesta ilustrada no validada previamente, la cual sería útil validar. Sin embargo, se hizo una prueba piloto con niños similares, socio-culturalmente, a los niños del estudio, y se modificó hasta lograr su comprensión por parte de los niños encuestados.

La información recolectada en este estudio habría sido más completa si hubiera incluido datos sobre el tipo de programas televisivos y la frecuencia de observación por parte de los niños del estudio

Agradecimientos. A los niños, directivas y profesores de la escuela Manuela Ayala de Gaitán, a Leonard Michael, Paola Andrea Rondón por su invaluable colaboración. También es importante destacar el valioso asesoramiento de los estadísticos María Nelcy Rodríguez y Milciades Ibáñez Pinilla.

\section{REFERENCIAS}

1. Aran S, Barata F, Busquet J, Medina P, Moron S. Infancia, violencia y televisión: usos televisivos y percepción infantil de la violencia en latelevisión. Barcelona: Trípodos; 2003.

2. Black D, Newman M. Television violence and children. BMJ.1995; 310(6975):273-4. 
3. Mares ML. (The Annenberg Public Policy Center of the University of Pennsylvania). Final report. Positive effects of Television on social behavior. A meta-analysis. Report Series. Number 3; June 17, 1996.

4. Piaget J. El desarrollo Mental del Niño. En: Piaget J. (Ed.) Seis Estudios de Psicología. Barcelona: Editorial Seix Barral, S. A.; 1971.

5. Bettelheim B. Psicoanálisis de los cuentos de hadas. Barcelona: Editorial Crítica; 2001.

6. Reyes- Gómez U, Sánchez NP, López G y Velasco MA. Violencia en las caricaturas: Análisis de dos series de televisión. Revista Mexicana de Puericultura y Pediatría. 1999;7(5): 22-8.

7. Lazar BA. Under the influence: An analysis of children's television regulation. Social Work.1994;39(1):67-74.

8. Delores M. The social learning theory [Internet] Tallahassee: Florida State University; 1998. Disponible en: http://www.criminology.fsu.edu/crimtheory/ bandura.htm. Consultado Dic 15, 2003.

9. Robinson TN, Wilde ML, Navracruz LC, Haydel KF, Varaday A. Effects of reducing children's television and video game use on aggressive behavior. Arch Pediatr Adolesc Med. 2001; 155(1):17-23.

10. Rushton JP. Positive effects of Television on social behavior. A meta-analysis. In: Mares ML. (The Annenberg Public Policy Center of the University of Pennsylvania). Final report. Report Series. Number 3; June 17, 1996.

11. Kremar M, Greene K. Predicting exposure to and uses of television violence. Journal of communication. 1999; 49(3): 24-45.

12. Cantor J, Nathanson AI. Children's fright reactions to television news. Journal of Communication. 1996; 46(4): 139-52.

13. Greenfield P, Beagles-Roos J. Radio vs. Television: Their cognitive impact on children of different socioeconomic and ethnic groups. Journal of communication. 1988; 38 (2): 71-92.

14. Strauss A, Corbin J. Bases de la investigación cualitativa, técnica y procedimientos para desarrollar la teoría fundamentada. Colombia: Editorial U. de Antioquia; 2002.

15. Mercado FJ y Torres TM. (Compiladores). Análisis cualitativo en salud. Teoría, Método y Práctica. México: Plaza y Valdés Editores; 2000.

16. Giacomini M, Cook, D. Users Guides to the medical literature XXIII. Qualitative research in health care A. Are the results of the study valid? JAMA, July 19, 2000; vol 284, N 3; 357-362

17. Giacomini M., Cook, D. Users Guides to the medical literature XXIII. Qualitative research in health care. B. what are the results and how do they help me care for my patients? JAMA, July 26, 2000; vol 284, N 4; 478-82.

18. Corman L. El Test del Dibujo de la Familia en la práctica médico - pedagógica. Buenos Aires: Editorial Kapelusz, S.A.; 1967.

19. Koppitz EM. El Dibujo de la Figura Humana en los Niños. Buenos Aires: Editorial Guadalupe; 1995.

20. Lewis R. Tests psicológicos y evaluación. México: Aiken Editorial Prentice Hall Hispanoamericana S.A.; 1996. 
21. León-Vásquez RL, Castañeda-Chang AM. [Internet] El Test del Dibujo de la Figura Humana De Koppitz en Niños Maltratados y no Maltratados. Disponible en: http://www.psicocentro.com/cgibin/articulo_s.asp?texto=art3a002. Consultado 18 de Enero de 2005.

22. Plubrukarn R, Theeramanoparp S. Human figure drawing test: validity in assessing intelligence in children aged 3-10 years. J Med Assoc Thai. 2003; Aug; 86 Suppl 3:S610-7.

23. Forensis 2001. Datos para la Vida. Instituto Nacional de Medicina Legal y Ciencias Forenses. Bogotá: Imprenta Nacional; 2002.

24. Gómez HA. La Violencia Necesita Estudios Epidemiológicos. Ponencia presentada al Primer Congreso Colombiano de Salud Pública, Medellín, Noviembre 1962. Tribuna Medica. 1962;Diciembre: 1-12.

25. Steen K, Hunskaar S. Gender and physical violence. Soc Sci Med. 2004; 59(3):567-71.

26. Anderson CA, Bushman BJ. Effects of Violent Video Games on Aggressive Behavior, Aggressive Cognition, Aggressive Affect, Physiological Arousal, and Prosocial Behavior: Meta-Analytic Review of the Scientific Literature. Psychol Sci. 2001 Sep;12(5):353-9.

27. Bushman BJ, Huesmann LR. Effects of televised violence on aggression. In: Singer DG, Singer JL Editores. Handbook of children and the media. Thousand Oaks, CA: Sage; 2001. p. 223-254.

28. Huesmann LR, Anderson CA, Berkowitz L, Donnerstein E, Johnson JD, Linz D,et al. Media violence influences on youth: Expert panel report to the U.S. Surgeon General. Institute for Social Research, University of Michigan, Ann Arbor; 2000.

29. Bandura A, Ribes-Inesta E. Analysis of Delinquency and Aggression. New Jersey: Lawrence Erlbaum Associates, INC; 1976.

30. Berkowitz L. Agresión: A Social Psychological Análisis. McGraw-Hill: San Francisco; 1962.

31. Centerwall B. Television and Violent Crime. New York: The Public Interest;1993.

32. Feshback S, Singer RD. Television and Aggression. San Francisco: Jossey-Bass; 1971.

33. American Psychiatric Association. Diagnostic and Statistical Manual of Mental Disorders, fourth Edition, (DSM IV). Washington, DC: American Psychiatric Association; 1995.

34. Shaywitz SE. Dyslexia. N Eng J Med. 1998; 338 (5): 307-312. 